\title{
Fourier-Transform Infrared Microspectroscopy (FT-IR) Study on Caput and Cauda Mouse Spermatozoa ${ }^{\dagger}$
}

\author{
Marianna Portaccio, Sonia Errico, Teresa Chioccarelli, Gilda Cobellis and Maria Lepore * \\ Dipartimento di Medicina Sperimentale, Università della Campania “L. Vanvitelli”, 80138 Naples, Italy; \\ marianna.portaccio@unicampania.it (M.P.); sonia.errico@unicampania.it (S.E.); \\ teresa.chioccarelli@unicampania.it (T.C.); gilda.cobellis@unicampania.it (G.C.) \\ * Correspondence: maria.lepore@unicampania.it \\ + Presented at the 6th International Electronic Conference on Sensors and Applications, \\ 15-30 November 2019; Available online: https://ecsa-6.sciforum.net/.
}

Published: 14 November 2019

\begin{abstract}
Fourier-Transform Infrared micro-spectroscopy ( $\mu$ FT-IR) was used for an in vitro investigation on spermatozoa (SPZ) samples separately collected from caput and cauda of mouse epididymis. SPZ are characterized by deep biochemical changes during the transit along the epididymis and they can constitute ideal candidates for a $\mu$ FT-IR investigation, thanks to the ability of this technique in analyzing cells at a molecular level. Appreciable differences were reported in the infrared spectra from caput and cauda SPZ, and biochemical changes in protein, nucleic acid, lipid, and carbohydrate content of cells were evidenced. The present investigation indicates that $\mu$ FT-IR can constitute a valuable tool for monitoring, in an easy and fast way, the changes suffered by SPZ during the transit along the epididymis.
\end{abstract}

Keywords: caput and cauda spermatozoa; $\mu$ FT-IR microspectroscopy; protein; nucleic acid; lipid and carbohydrate components

\section{Introduction}

Fourier-Transform IR (FT-IR) spectroscopic technique is nowadays a very valuable tool for the nondestructive analysis of biological specimens. FT-IR results are also particularly useful in cytological and histological diagnosis by using imaging procedures [1]. FT-IR is able to reveal bonds presenting a change of dipole moment during vibrational motion. These vibrational modes can be quantitatively characterized by IR spectroscopy, providing a very sensitive, label-free tool for studying molecular composition and dynamics without perturbing the sample. When FT-IR spectroscopy is performed on biological materials, the most important spectral regions are the fingerprint region (600-1450 $\mathrm{cm}^{-1}$ ) and the amide I and amide II (amide I/II) region (1500-1700 $\mathrm{cm}^{-1}$ ). The higher-wavenumber region $\left(2800-3500 \mathrm{~cm}^{-1}\right)$ is due to stretching vibrations such as $\mathrm{S}-\mathrm{H}, \mathrm{C}-\mathrm{H}$, $\mathrm{N}-\mathrm{H}$ and $\mathrm{O}-\mathrm{H}$, whereas the lower-wavenumber regions typically correspond to bending and carbon skeleton fingerprint vibrations. These regions are able to give a complete biochemical fingerprint of the structure and function of investigated samples and have been adopted in a very large number of different samples of biological interest.

Recently, FT-IR spectroscopy has been adopted for investigating spermatozoa (SPZ) samples in order to principally take advantage of its non-invasivity. In particular, cyanide toxicity effects on rat sperm have been investigated at a molecular level by using FT-IR technique. Cyanide compounds are largely used in industries and are considered to be a ubiquitous pollutant in the environment. 
The use of FT-IR spectroscopy has also allowed an investigation on the secondary structure of protein components. It is worth noting that a complementary vibrational technique, the Raman technique, has been used for investigating oxidative DNA damage in human sperm [2].

In the present paper, FT-IR microspectroscopy ( $\mu$ FT-IR) has been adopted for characterizing SPZ separately collected from caput and cauda of the epididymis. Interestingly, SPZ are transcriptionally and translationally silent cells. During the epididymal transit, from caput-to-cauda, these cells undergo maturation via massive biochemical changes [3,4] that include: (i) decreases of cholesterol; (ii) qualitative composition of cytoplasmic proteins and RNA; (iii) protein degradation and phosphorylation; (iv) acquisition of GPI-anchored membrane proteins; (v) proteolytic remodelling of membrane proteins; (vi) fatty acid composition from saturated to polyunsaturated forms; (vii) DNA methylation; and (viii) inter/intra protamine disulfide-bound formation. Due to their remarkable biochemical differences, caput and cauda SPZ can be considered ideal candidates for an FT-IR spectroscopy investigation.

In this report, we summarize the preliminary results of an investigation on mouse sperm samples from caput and cauda epididymis. To our knowledge, this is the first time that vibrational spectroscopies are adopted for investigating the changes occurring in SPZ during the epididymal transit.

\section{Materials and Methods}

\subsection{Collection of Sperm Samples from Caput and Cauda Epididymis}

Adult male mice (CD1 background, from Charles River Laboratories, Lecco, Italy) were euthanized by asphyxia with $\mathrm{CO}_{2}$ and epididymis were dissected for sperm collection. Briefly, caput and cauda epididymis were separately immersed in PBS (pH 7.6) and slightly cut to endorse sperm extrusion from the epididymal duct, as previously reported [5]. Spermatozoa samples, separately collected from caput and cauda epididymis, were filtered throughout cheesecloth, washed by centrifugation (200× $g$ for $5 \mathrm{~min}$ ), in $0.9 \% \mathrm{NaCl}$ solution, and pellets were processed for FT-IR analysis.

All animal studies were carried out in accordance with the principles and procedures outlined in the National Institute of Health Guide for Care and Use of Laboratory Animals and approved by the Italian Ministry of Research and the Italian Ministry of Health.

\subsection{FT-IR Spectral Analysis}

For FT-IR spectral analysis, SPZ pellets were resuspended in $0.9 \% \mathrm{NaCl}$ solution and drops of a few microliters were used for spectra acquisition. For this purpose, a Perkin Elmer Spectrum One FT-IR spectrometer equipped with a Perkin Elmer Multiscope system infrared microscope (Mercury Cadmium Telluride detector, Perkin Elmer Inc., Hopkinton, MA, USA) was used. Spectral acquisitions were performed in specular-reflection mode with thin layers of samples $(2 \mathrm{~L})$ put on metallic IR-reflective surface. The background spectrum was collected from the metallic IR-reflective surface without sample. A sampling spot on the surface was selected through an objective (10× optical or $15 \times$ infrared). All spectra were collected using 64 scans in the range from 4000 to $600 \mathrm{~cm}^{-1}$ with a $4 \mathrm{~cm}^{-1}$ spectral resolution.

The spectra were preliminarily analyzed using the application routines provided by the software package ("Spectrum" User Guide, Perkin Elmer Inc., Hopkinton, MA, USA) controlling the whole data acquisition system. "Spectrum" (release 5.0.2, 2004) is the main Perkin Elmer software package for collecting, viewing and processing IR spectra [6].

\section{Results and Discussion}

The $\mu$ FT-IR average spectra of mouse SPZ samples from caput and cauda epididymis (caput and cauda SPZ) were obtained in the $4000-600 \mathrm{~cm}^{-1}$ range. The peak position and band assignments were done in agreement with [6]. In order to evidence the differences occurring between the two spectra, they were divided into four wavenumber ranges: $3600-3000 \mathrm{~cm}^{-1}$ (Figure 1), 3050-2800 $\mathrm{cm}^{-1}$ (Figure 2), 1800-1200 cm-1 (Figure 3) and 1200-600 $\mathrm{cm}^{-1}$ (Figure 4). 
Figure 1 shows the average $\mu$ FT-IR spectra of caput and cauda SPZ in the range of $3600-3000 \mathrm{~cm}^{-1}$. In this range, there is the large contribution of amide A band (located at 3309 and 3290 for caput and cauda SPZ, respectively) due to $\mathrm{N}-\mathrm{H}$ and $\mathrm{O}-\mathrm{H}$ stretching modes of proteins and intermolecular $\mathrm{H}$ bonding. It is worth noting that the shape of this band changes between caput and cauda samples. In particular, for caput SPZ, the maximum is located at $\sim 3400 \mathrm{~cm}^{-1}$; conversely, for cauda SPZ, the maximum is located at $3290 \mathrm{~cm}^{-1}$. A very weak contribution $3070 \mathrm{~cm}^{-1}$ can be noticed only in the case of cauda samples and can be attributed to the N-H stretching of amide B. In order to analyze the changes in the lipid content of caput and cauda SPZ, the spectral region from 3050 to $2800 \mathrm{~cm}^{-1}$ was considered (Figure 2). In this region, it is possible to evidence the contributions of $\mathrm{CH}_{3}$ asymmetric stretching (located at 2957 and $2959 \mathrm{~cm}^{-1}$ for caput and cauda samples, respectively), $\mathrm{CH}_{2}$ asymmetric stretching (located at 2922 and $2924 \mathrm{~cm}^{-1}$ for caput and cauda samples, respectively), $\mathrm{CH}_{3}$ symmetric stretching (located at $2875 \mathrm{~cm}^{-1}$ for both caput and cauda samples) and $\mathrm{CH}_{2}$ symmetric stretching (located at 2851 and $2853 \mathrm{~cm}^{-1}$ for caput and cauda samples, respectively). It is important to note that the two spectra are largely different in shape and relative intensity of the main peaks, even though the position of the different $\mathrm{CH}_{2}$ and $\mathrm{CH}_{3}$ contribution does not change in a crucial way. In particular, the position of $\mathrm{CH}_{2}$ symmetric stretching does not show significant changes, taking into account the spectral resolution of our equipment $\left(4 \mathrm{~cm}^{-1}\right)$. This can indicate that no important changes in membrane fluidity occur [7]. In this region of the spectra, a contribution at $3013 \mathrm{~cm}^{-1}$ is also present which is more evident for caput SPZ samples. This band is usually assigned to an olefinic functional group in unsaturated lipids, mainly due to $\mathrm{C}-\mathrm{H}$ stretching mode. This band can be used for further investigating lipid peroxidation in tissue [8].

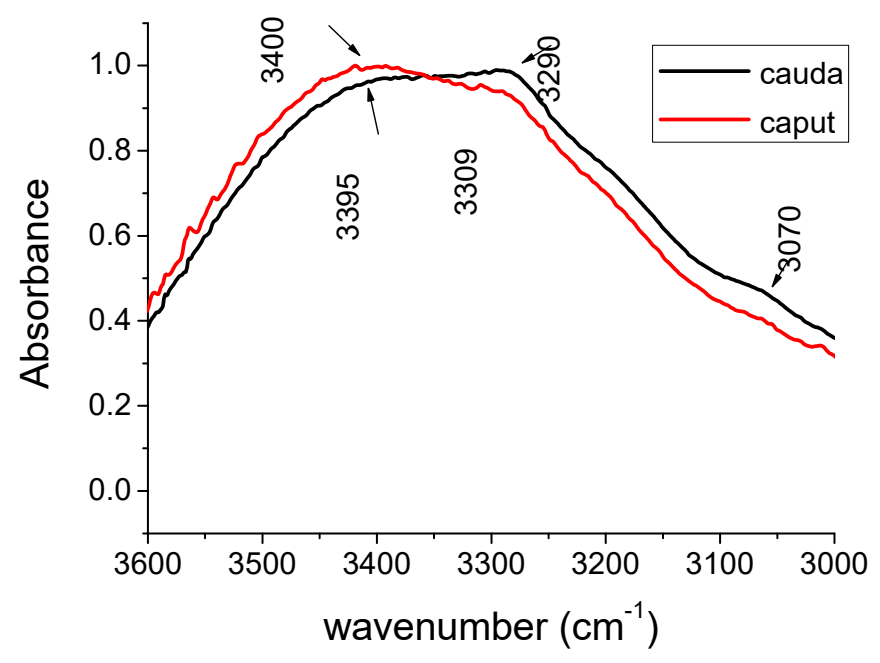

Figure 1. FT-IR spectra of caput and cauda sperm of WT mouse in $3600-3000 \mathrm{~cm}^{-1}$ region. 


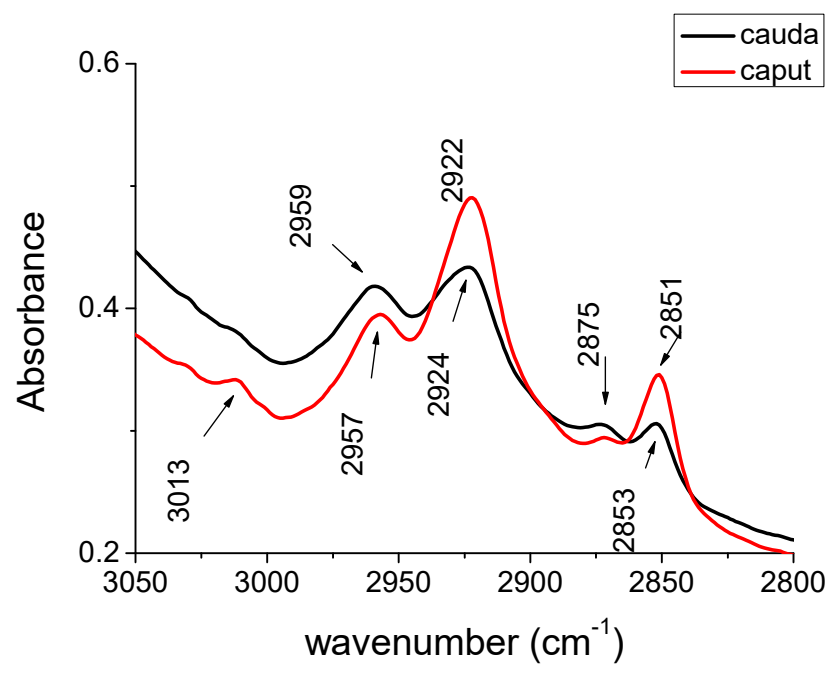

Figure 2. FT-IR spectra of caput and cauda sperm of WT mouse in 3050-2800 cm-1 region.

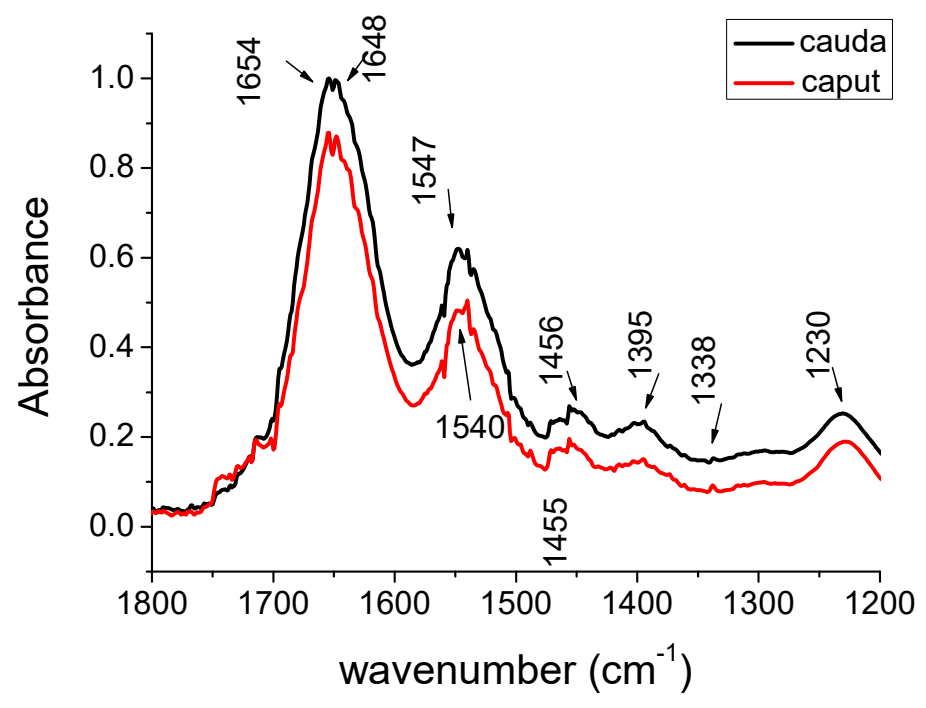

Figure 3. FT-IR spectra of caput and cauda sperm of WT mouse in $1800-1200 \mathrm{~cm}^{-1}$ region.

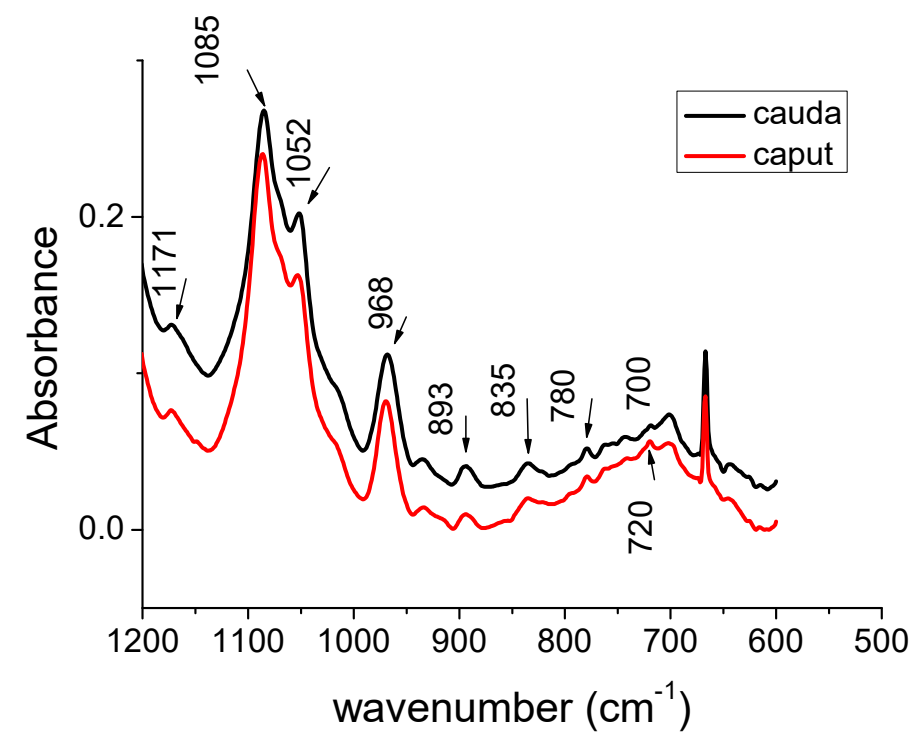

Figure 4. FT-IR spectra of caput and cauda sperm of WT mouse in $1200-600 \mathrm{~cm}^{-1}$ region. 
In Figure 3, the $1800-1200 \mathrm{~cm}^{-1}$ region of $\mu$ FT-IR spectra of caput and cauda SPZ is reported. In this region, the main contributions are due to protein content. In particular, the band due to the Amide I C $=\mathrm{O}$ stretching is positioned $\sim 1650 \mathrm{~cm}^{-1}$ for the two different samples; however, differences can be noted in the band's shape. In Figure 3, the contributions of Amide II (at 1540 and $1547 \mathrm{~cm}^{-1}$ for caput and cauda samples, respectively) and Amide III (at $1300 \mathrm{~cm}^{-1}$ for both caput and cauda samples) are present. The small band positioned at 1455 and $1456 \mathrm{~cm}^{-1}$ for caput and cauda samples, respectively, can be due to $\mathrm{CH}_{3}$ asymmetric bending and $\mathrm{CH}_{2}$ scissoring mode that can be associated with lipid and protein contents. At $1395 \mathrm{~cm}^{-1}$, a small feature can be noticed and it can be attributed to COO- symmetric stretching vibration mode related to fatty acids. The band located at $1230 \mathrm{~cm}^{-1}$ is due to $\mathrm{PO}_{2}^{-}$asymmetric stretching mode related to nucleic acids and phospholipids contributions.

In Figure 4, the region of short wavenumber $\left(1200-600 \mathrm{~cm}^{-1}\right)$ of $\mu$ FT-IR spectra of caput and cauda SPZ is shown. This region is mainly related to DNA contributions. The most important ones are located at 1085 ( $\mathrm{PO}_{2-}^{-}$symmetric stretching) and $1052 \mathrm{~cm}^{-1}$ (C-O stretching). In this region too, differences can be noted in the band shape. Additionally, the other structures positioned at $968 \mathrm{~cm}^{-1}$ (C-C stretching), $893 \mathrm{~cm}^{-1}$ (deoxyribose ring), $835 \mathrm{~cm}^{-1}$ (S-type sugar) are attributed to DNA. A complete list of the peak position and band assignments is reported in Table 1.

Table 1. Mode assignments for the FT-IR frequency values in caput and cauda sperm samples, abbreviation: as $=$ asymmetric, $\mathrm{s}=$ symmetric, $v=$ stretching, $\mathrm{vbr}=$ vibration, $\mathrm{b}=$ bending, scis $=$ scissoring.

\begin{tabular}{|c|c|c|c|}
\hline $\begin{array}{l}\text { Peak caput } \\
\qquad \mathrm{cm}^{-1}\end{array}$ & $\begin{array}{l}\text { Peak cauda } \\
\qquad \mathrm{cm}^{-1}\end{array}$ & Peak Assignments & Biochemical Molecules \\
\hline 3400 & 3395 & Hydrogen bonded O-H N-H & protein water \\
\hline 3309 & 3290 & Amide A N-H & protein \\
\hline 3013 & 3013 & olefinic $=\mathrm{C}-\mathrm{H}$ & unsaturated lipid \\
\hline 2957 & 2959 & $\mathrm{CH}_{3}$ as & lipid \\
\hline 2922 & 2924 & $\mathrm{CH}_{2}$ as & lipid \\
\hline 2875 & 2875 & $\mathrm{CH}_{3} \mathrm{~S}$ & lipid \\
\hline 2851 & 2853 & $\mathrm{CH}_{2} \mathrm{~s}$ & lipid \\
\hline 1654 & 1654 & Amide I-helix & protein \\
\hline 1648 & 1648 & Amide I & protein \\
\hline 1540 & 1547 & Amide II & protein \\
\hline 1455 & 1456 & $\begin{array}{l}\mathrm{CH}_{3} \text { as b } \\
\mathrm{CH}_{2} \text { scis }\end{array}$ & lipid and protein \\
\hline 1395 & 1395 & COO- s & fatty acid \\
\hline 1300 & 1300 & Amide III & protein \\
\hline 1230 & 1230 & $\mathrm{PO}_{2}$ - as & $\begin{array}{l}\text { nucleic acids and } \\
\text { phospholipids }\end{array}$ \\
\hline 1171 & 1171 & $\mathrm{CO}-\mathrm{O}-\mathrm{C}$ & glycogen \\
\hline 1085 & 1085 & $\mathrm{PO}_{2-\mathrm{S}}$ & nucleic acids \\
\hline 1052 & 1052 & $\mathrm{C}-\mathrm{O}$ & $\begin{array}{c}\text { saccharides and DNA } \\
\text { deoxyribose }\end{array}$ \\
\hline 968 & 968 & $\mathrm{C}-\mathrm{C}$ & DNA backbone \\
\hline 893 & 893 & Deoxyribose ring & DNA \\
\hline 835 & 835 & DNA S-type sugar & DNA \\
\hline
\end{tabular}

\section{Conclusions}

The results reported here indicate that $\mu$ FT-IR spectroscopy can constitute a useful tool for investigated changes occurring in SPZ from caput and cauda epididymis of mice in a rapid and easy way. Biochemical changes in protein, nucleic acid, lipid, and carbohydrate content of cells have been evidenced. Further work is in progress in order to quantitatively assess the observed differences. 
Acknowledgments: This work was supported by Università della Campania "L. Vanvitelli" (Italy) under the project "Ricerca Scientifica di Dipartimento" (2017). The authors are pleased to thank Nadia Diano for useful discussions.

Conflicts of Interest: The authors declare that there is no conflict of interest.

Compliance with Ethical Standards: All applicable international, national, and/or institutional guidelines for the care and use of animals were followed.

\section{References}

1. Baker, M.J.; Trevisan, J.; Bassan, P.; Bhargava, R.; Butler, H.J.; Dorling, K.M.; Fielden, P.R.; Fogarty, S.W.; Fullwood, N.J.; Heys, K.A.; et al. Using Fourier transform IR spectroscopy to analyze biological materials. Nat. Protoc. 2014, 9, 1771-1791.

2. Shivanoor, S.M.; David, M. Fourier transform infrared (FT-IR) study on cyanide induced biochemical and structural changes in rat sperm. Toxicol. Rep. 2015, 2, 1347-1356.

3. Sánchez, V.; Redmann, K.; Wistuba, J.; Wübbeling, F.; Burger, M.; Oldenhof, H.; Wolkers, W.F.; Kliesch, S.; Schlatt, S.; Mallidis, C. Oxidative DNA damage in human sperm can be detected by Raman microspectroscopy. Fertil. Steril. 2012, 98, 1124-1129.

4. Marciani, S.; Tamburrino, L.; Muratori, M.; Baldi, E. Epididymal sperm transport and fertilization. In Endocrinology of the Testis and Male Reproduction Endocrinology 1; Simoni, M., Huhtaniemi, I.T., Eds.; Springer International Publishing AG: Berlin/Heidelberg, Germany, 2017; pp. 457-478.

5. Cooper, T.G. Epididymis. In Enciclopedia of Reproduction; Knobil, E., Neill, J.D., Eds.; Academic Press: Cambridge, MA, USA, 2003; pp. 1-17.

6. Portaccio, M.; d'Apuzzo, F.; Perillo, L.; Grassia, V.; Errico, S.; Lepore, M. Infrared microspectroscopy characterization of gingival crevicular fluid during orthodontic treatment. J. Mol. Struct. 2019, 1176, 847-854.

7. Liu, K.Z.; Schultz, C.P.; Mohammad, R.M.; Johnston, J.B.; Mantsch, H.H. Infrared spectroscopic study of bryostatin 1-induced membrane alterations in a B-CLLcell line. Leukemia 1999, 13, 1273-1280.

8. Severcan, F.; Gorgulu, G.; Gorgulu, S.T.; Guray, T. Rapid monitoring of diabetes-induced lipid peroxidation by Fourier transform infrared spectroscopy: Evidence from rat liver microsomal membranes. Anal. Biochem. 2005, 339, 36-40.

(C) 2019 by the authors. Licensee MDPI, Basel, Switzerland. This article is an open access article distributed under the terms and conditions of the Creative Commons Attribution (CC BY) license (http://creativecommons.org/licenses/by/4.0/). 